\title{
Bloqueo de nervios periféricos guiados por ecografía en pacientes con hemofilia
}

\section{Ultrasound-guided peripheral nerve blocks in hemophilia with patients}

\author{
Ana Laura Douglas Price PhD. ${ }^{1, a,{ }^{*},}$, Horacio Alberto Caviglia MD. ${ }^{1, a}$, Edith Negronida ${ }^{1, b}$, Melina Machado ${ }^{1, b}$, \\ María Constanza Cannizzaro ${ }^{1, b}$ \\ Hospital General de Agudos Juan A. Fernández. Buenos Aires, Argentina. \\ Servicio de Ortopedia y Traumatología. \\ Servicio de Anestesia.
}

Los autores declaran no tener ningún conflicto de intereses.

Fecha de recepción: 04 de marzo de 2021 / Fecha de aceptación: 10 de abril de 2021

\begin{abstract}
Introduction: Hemophilia is a coagulation disorder; it is a recessive disease linked to the $\mathrm{X}$ chromosome. In patients with hemophilia (PWH), regional anesthetic blocks have been considered a contraindication. Safety has been increased by performing them guided by Ultrasound. The objective of our work is to show our experience in PWH and peripheral nerve blocks. Material and Method: 41 PWH were operated under regional analgesia with Ultrasound-Guided Peripheral Nerve Blocks associated with general anesthesia in the period 2006-2019. All patients were Hemophilia A. Three patients had inhibitors. The mean age was 35 years. 40 lower limb blocks and 2 upper limb blocks were performed. The Sonosite ${ }^{\circledR}$ equipment model Micromaxx was used. Results: All patients presented adequate peripheral nerve block for an average time of 12.5 hours (8-24). There were no complications. Conclusion: The present study shows that Ultrasound-Guided Peripheral Nerve Blocks in PCH is a safe procedure, which reduces the requirements of opioids and the side effects of them, improving the postoperative period and the recovery of patients.
\end{abstract}

Key words: Ultrasound, haemophilia, peripheral nerve block.

\section{RESUMEN}

Introducción: La hemofilia es un trastorno de la coagulación, es una enfermedad recesiva ligada al cromosoma X. En pacientes con hemofilia (PCH) los bloqueos regionales anestésicos se han considerado una contraindicación. Se ha aumentado la seguridad realizándolos guiados por Ecografía. El objetivo de nuestro trabajo es mostrar nuestra experiencia en PCH y bloqueos de nervios periféricos. Material y Método: 41 PCH fueron operados bajo analgesia regional con Bloqueos de Nervios Periféricos Guiados por Ecografía asociado a la anestesia general en el período 2006-2019. Todos los pacientes eran hemofilia A. Tres pacientes presentaban inhibidores. La edad media fue de 35 años. Se realizaron 40 bloqueos de miembros inferiores y 2 bloqueos miembros superiores. Se utilizó el equipo Sonosite ${ }^{\circledR}$ modelo Micromaxx. Resultados: Todos los pacientes presentaron adecuado bloqueo de nervio periférico durante un tiempo promedio de 12,5 h (8-24). No se presentaron complicaciones. Conclusión: El presente estudio muestra que los Bloqueos de Nervios Periféricos Guiados por Ecografía en PCH es un procedimiento seguro, que reduce los requisitos de los opioides y los efectos secundarios de ellos, mejorando el posoperatorio y la recuperación de los pacientes.

Palabras clave: Ecografía, hemofilia, bloqueos nervios periféricos. 


\section{Introducción}

a hemofilia es un trastorno de la coagulación, es una enfermedad recesiva ligada al cromosoma X. La hemofilia A presenta una deficiencia hereditaria del factor de coagulación VIII (FVIII) y la hemofilia B la del factor IX dependiente de la vitamina $K$ (FIX), respectivamente[1]. De acuerdo a la última encuesta Mundial de la Federación Mundial de la hemofilia 337.641 personas padecen trastornos de la coagulación, el $62 \%$ padece hemofilia A, el 10\% Hemofilia B y el $0,7 \%$ de hemofilia que no se puede tipificar[2]

En pacientes con hemofilia ( $\mathrm{PCH}$ ) los bloqueos regionales anestésicos se han considerado una contraindicación[3]. Se ha aumentado la seguridad debido a una mejor monitorización de la anatomía y la colocación de la aguja de los bloqueos de nervios periféricos guiados por ecografía (BNPGE), lo cual es importante en los PCH con la corrección adecuada de los factores de coagulación. En la literatura hemos encontrado solo algunos pocos reportes mostrando la experiencia de los bloqueos de nervios periféricos en los miembros inferiores[3],[4].

El objetivo de nuestro trabajo es mostrar nuestra experiencia en PCH y BNPGE tanto en miembros superiores como inferiores.

\section{Material y Método}

Cuarenta y un PCH fueron operados bajo analgesia regional con BNPGE asociado a la anestesia general en el período 20062019. Todos los pacientes firmaron el consentimiento informado.

Todos los pacientes eran hemofilia A, 3 eran moderados y 38 severos. Tres pacientes presentaban inhibidores. La edad media fue de 35 años (9-62). Se realizaron 21 bloqueos del nervio femoral, 18 bloqueos del nervio ciático, 1 bloqueo del nervio safeno y 2 bloqueos supraclaviculares. A un paciente se le realizó bloqueo femoral y ciático y a otro paciente un bloqueo ciático y safeno.

A los pacientes se les realizó la adecuada corrección de los factores de la coagulación y posteriormente se les realizó el BNPGE. Se utilizó el equipo Sonosite ${ }^{\circledR}$ modelo Micromaxx. Todos los procedimientos fueron realizados en la sala de recuperación del área quirúrgica de nuestra institución. Se utilizó para todos los bloqueos una mezcla al $50 \%$ de cada una de bupivacaína 0,25\% y de lidocaína con epinefrina al 1,5\%, dependiendo del nervio a bloquear fue el volumen utilizado. A todos los pacientes se les realizó el procedimiento en condiciones estériles, se realizó la preparación de la piel en dos tiempos con descontaminación de la superficie y luego el embrocado con solución de iodopovidona. Se utilizó un transductor de alta frecuencia (5-13 MHz) que fue cubierto con un cobertor estéril y gel para ultrasonido también estéril.

\section{Técnicas utilizadas para cada bloqueo}

Bloqueo del nervio femoral: Se realizó lateral a la arteria femoral dentro del ligamento inguinal. La arteria femoral se identifica debajo del ligamento inguinal con el transductor en el eje transversal al recorrido longitudinal del nervio, donde aún no se ha dividido la arteria. Este es el sitio óptimo para el blo- queo del nervio femoral. El músculo iliopsoas se encuentra lateral y profundo a la arteria femoral y cubierto por la fascia ilíaca que se ve hiperecoica. Profundo a esta fascia ilíaca y lateral a la arteria femoral encontramos el nervio femoral que lo vemos triangular e hiperecoico. La aguja se insertó en el borde lateral del transductor en un ángulo que se obtenga un "enfoque en el plano" hacia el nervio femoral[5]. Se utilizó dependiendo de la contextura del paciente agujas Pajunk ${ }^{\circledR}$ Sonoplex Stim de 50 o $100 \mathrm{~mm} * 21 \mathrm{G}$. El anestésico se colocó por encima y por debajo del nervio para maximizar la cobertura del mismo, el volumen utilizado fue de $20 \mathrm{ml}$.

Bloqueo del nervio ciático: El procedimiento se realizó con el paciente en posición de decúbito dorsal. El nervio ciático está compuesto por dos componentes mayores, el nervio tibial y el tronco del nervio peroneo común. Estos dos nervios independientes no comparten sus fibras, pero si el trayecto común hasta que se dividen en la fosa poplítea. Cuando se realiza el rastreo ecográfico en el hueco poplíteo, el nervio ciático se encuentra en el ápice de la fosa poplítea (bordeado lateralmente por las cabezas largas y cortas de los músculos bíceps femoral y medialmente por los músculos semimembranoso-semitendinoso) y dorsal (superficial) a la vena y arteria poplítea. El rastreo de ecografía en el eje corto correspondiente del nervio ciático se obtiene colocando el transductor en una orientación axial sobre el vértice de la fosa poplítea. El nervio ciático aparece como una estructura polifascicular hiperecoica redonda dorsal a la arteria y vena poplíteas. La imagen ecográfica de eje corto correspondiente de la bifurcación del nervio ciático se obtiene moviendo el transductor distalmente hasta que el nervio tibial situado medialmente y el nervio peroneo común situado lateralmente se separan físicamente entre sí. Este es el lugar donde se realizó la inyección de los anestésicos locales[6].

Se realizó la inyección lateral al nervio, en el punto más proximal donde aún no se ha dividido en los dos nervios, de esta forma se puede alcanzar a los dos componentes y asegurar la analgesia completa infrapoplítea, se utilizó un volumen de 30 $\mathrm{ml}$. Se utilizó una aguja Pajunk ${ }^{\circledR}$ Sonoplex Stim de 50 o 100 mm *21 G.

Bloqueo del nervio safeno: El paciente se colocó en posición de decúbito supino. El bloqueo del nervio safeno fue realizado a nivel de la mitad del muslo en el canal del aductor o debajo de la rodilla dependiendo del sitio que se deseaba la anestesia. El transductor se colocó perpendicular al eje longitudinal del miembro inferior, 5 a $7 \mathrm{~cm}$ proximal al hueco poplíteo y se desliza proximalmente hasta que la imagen hiperecoica de la fascia del aductor desaparece y se identifica la arteria femoral. El nervio safeno se identifica con una imagen circular hiperecoica anterolateral a la arteria femoral. El mismo fue realizado usando una aguja Pajunk ${ }^{\circledR}$ Sonoplex Stim de 50 mm 21G, el volumen utilizado para este nervio fue de $10 \mathrm{ml}$.[7]-[10] Cuando la anestesia requerida era para realizar un procedimiento en la pierna y el pie el bloqueo del nervio safeno se realizó por debajo de la rodilla. El transductor se colocó a nivel de la tuberosidad tibial, la vista de la vena safena y la fascia lata en un eje corto y se realiza la infiltración entre estas dos estructuras[11].

Bloqueo supraclavicular: Este bloqueo se realiza donde el plexo braquial se presenta de forma más compacta, en el nivel de la división de los troncos primarios del mismo justo por encima de la clavícula[12]. Debido a esta característica anatómica el bloqueo proporciona una latencia corta y una anestesia 
Tabla 1. Resultados de bloqueos de nervios periféricos en PCH guiados por ecografía

\begin{tabular}{|c|c|c|c|c|c|c|c|}
\hline Paciente & Cirugía & BNPGE & Edad & $\begin{array}{c}\text { Tipo de } \\
\text { hemofilia }\end{array}$ & Grado & Inhibidor & $\begin{array}{l}\text { Duración del } \\
\text { bloqueo }\end{array}$ \\
\hline 1 & ATR & Femoral & 38 & A & Severa & No & 12 \\
\hline 2 & ATR & Femoral/ciático & 30 & A & Severa & No & 8 \\
\hline 3 & ATR & Femoral & 39 & A & Severa & No & 24 \\
\hline 4 & ATR & Femoral & 54 & A & Severa & No & 16 \\
\hline 5 & ATR & Ciático & 55 & A & Severa & No & 13 \\
\hline 6 & Artroplastía de tobillo & Femoral & 44 & A & Severa & No & 9 \\
\hline 7 & tenotomía de flexores de cadera & Ciático & 12 & A & Severa & No & 15 \\
\hline 8 & ATR & Femoral & 62 & A & Moderada & No & 16 \\
\hline 9 & Artroscopía de codo & Supraclavicular & 19 & A & Severa & No & 18 \\
\hline 10 & ATR & Femoral & 39 & A & Severa & No & 12 \\
\hline 11 & Osteotomía de tibia distal & Ciático & 35 & A & Severa & No & 21 \\
\hline 12 & Artroscopía de tobillo & Ciático & 34 & A & Severa & No & 12 \\
\hline 13 & Tenotomía de flexores de cadera & Femoral & 9 & A & Severa & Si & 8 \\
\hline 14 & ATR & Femoral & 36 & A & Moderada & No & 18 \\
\hline 15 & ATR & Femoral & 52 & A & Severa & No & 7 \\
\hline 16 & Osteotomía de rodilla & Ciático & 23 & A & Severa & No & 12 \\
\hline 17 & ATR & Femoral & 46 & A & Severa & No & 12 \\
\hline 18 & Biopsia de rodilla & Femoral & 37 & A & Severa & No & 9 \\
\hline 19 & Reconstrucción femoropatelar & Femoral & 15 & A & Severa & No & 22 \\
\hline 20 & Artroplastía de tobillo & Ciático/safeno & 62 & A & Moderada & No & 13 \\
\hline 21 & Pseudotumor de rodilla & Ciático & 40 & A & Severa & $\mathrm{Si}$ & 23 \\
\hline 22 & Biopsia de rodilla & Ciático & 38 & A & Severa & Si & 16 \\
\hline 23 & Osteotomía de pie & Femoral & 17 & A & Severa & No & 8 \\
\hline 24 & Liberación del Aquiles & Ciático & 17 & A & SEVERA & No & 11 \\
\hline 25 & ATR & Femoral & 37 & A & Severa & No & 9 \\
\hline 26 & ATR & Femoral & 29 & A & Severa & No & 15 \\
\hline 27 & Osteosíntesis de fémur & Femoral & 16 & A & Severa & No & 12 \\
\hline 28 & ATR & Femoral & 55 & A & Severa & No & 9 \\
\hline 29 & Artroscopía de tobillo & Ciático & 37 & A & Severa & No & 11 \\
\hline 30 & ATR & Femoral & 40 & A & Severa & No & 10 \\
\hline 31 & Liberación nervio cubital & Supraclavicular & 43 & A & Severa & No & 9 \\
\hline 32 & Artroscopía de tobillo & Ciático & 36 & A & Severa & No & 10 \\
\hline 33 & ATR & Femoral & 50 & A & Severa & No & 6 \\
\hline 34 & Pseudotumorde rodilla & Ciático & 26 & A & Severa & No & 13 \\
\hline 35 & ATR & Femoral & 54 & A & Severa & No & 11 \\
\hline 36 & Tenotomía de flexores de cadera & Ciático & 38 & A & Severa & No & 9 \\
\hline 37 & Biopsia de tobillo & Femoral & 28 & A & Severa & No & 15 \\
\hline 38 & Liberación del Aquiles & Ciático & 16 & A & Severa & No & 10 \\
\hline 39 & Artroscopía de tobillo & Ciático & 30 & A & Severa & No & 8 \\
\hline 40 & Tenotomía de flexores de cadera & Ciático & 16 & A & Severa & No & 9 \\
\hline 41 & Artroscopía de tobillo & Ciático & 25 & A & Severa & No & 10 \\
\hline
\end{tabular}


completa y confiable para las cirugías del miembro superior. La vista del ultrasonido clásica muestra a los troncos primarios del plexo braquial hipoecoicos agrupados lateralmente a la arteria subclavia y sobre la primera costilla, que proyecta una sombra acústica cuando el haz de ultrasonido es atenuado por el hueso[13]. Según las características anatómicas del paciente se utilizó una aguja Pajunk ${ }^{\circledR}$ Sonoplex Stim de 50 mm 21G, se utilizó un volumen de $20 \mathrm{ml}$.

\section{Resultados}

En la Tabla 1 se muestran los datos de todos los pacientes a los que se les realizó BNPGE (procedimiento realizado, edad, tipo de bloqueo, tipo de hemofilia y la presencia o no de inhibidores). La edad promedio fue de 35 años (9-62). Todos los pacientes presentaron adecuado bloqueo de nervio periférico durante un tiempo promedio de $12,5 \mathrm{~h}$ (8-24). No se presentaron complicaciones de sangrado ni de infecciones, a nivel del bloqueo supraclavicular no se presentó la complicación más frecuente de esta que es el neumotórax[14].

\section{Discusión}

El uso de analgesia neuroaxial puede ser ventajoso para la recuperación reduciendo el uso de los efectos adversos de los opioides[3],[4]. La realización de bloqueos neuroaxiales, ya sea espinal o epidural en los pacientes con problemas de la coagulación, no solo los PCH, debido al riesgo potencial de la formación de un hematoma en un espacio cerrado como lo es el conducto espinal pudiendo producir compresión de la médula y el consiguiente déficit neurológico se encuentran contraindicados[15].

Los BNPGE reducen el uso de los opioides y los efectos secundarios de estos, tampoco presentan las complicaciones de los bloqueos neuroaxiales centrales como la punción dural, la formación de hematomas y el efecto de bloqueo motor en ambas piernas[3],[16]. También reduce los requerimientos de analgésicos sistémicos durante el período de efecto del bloqueo del nervio periférico que en nuestra casuística es de 12,5 h en promedio. La técnica de ecografía mejora la tasa de éxito y reduce aún más los riesgos de lesiones nerviosas y también de punción vascular debido a que se puede observar el vaso durante el procedimiento[4].

Los bloqueos de nervios periféricos pueden tener complicaciones también; de estos se conoce que pueden no proveer la analgesia esperada, la toxicidad del anestésico local pudiendo producir lesiones a nivel de los nervios involucrados y la formación de hematomas con el consecuente daño del o los nervios que se intentan bloquear[17]. Nosotros no tuvimos casos de complicaciones relacionadas al procedimiento ni al uso de los anestésicos pero hay que tenerlo en cuenta, pero creemos que los beneficios del bloqueo son muy importantes.

Como mencionamos previamente en nuestros pacientes evidenciamos que en un promedio de $12,5 \mathrm{~h}$ no requirieron tratamiento para el dolor. Si bien los opioides son efectivos para el tratamiento del dolor presentan efectos secundarios como la depresión respiratoria que en el manejo posoperatorio en una sala de recuperación puede ser un problema importante, así como también prurito, náuseas, vómitos, retención urinaria, íleo paralítico que dificultan la movilización temprana y la rápida recuperación[18].

En nuestro caso utilizamos para realizar el bloqueo el rastreo ecográfico asociado a la utilización del Doppler para reducir las posibles complicaciones asociadas a la punción de los vasos de la región. También utilizamos una aguja con bisel corto en pos de reducir aún más los riesgos.

Si bien en nuestro trabajo no medimos los niveles de concentración de factor durante todo el procedimiento (preoperatorio, durante la cirugía y el posoperatorio) los pacientes se benefician debido a que los niveles de factores se encuentran estables durante el período quirúrgico y en el posoperatorio hasta los 7 días como mencionaron Vanarase y colaboradores en una revisión de 21 artroplastias de rodilla en 15 PCH. En este trabajo los bloqueos para nervios periféricos fueron utilizados para el manejo del dolor posoperatorio no para el procedimiento. Determinaron los valores de factor preoperatorio y una hora antes de la cirugía y estos valores se mantuvieron al 100\% durante la cirugía. Al realizar el seguimiento posoperatorio de la concentración de factores evidenciaron que estos se mantenían al 50\% por 7 a 10 días[4]. Esto es un beneficio directo para el paciente y también en el costo beneficio relacionado a los gastos de la salud.

En nuestra casuística tuvimos 3 pacientes que presentaban inhibidor, pero eso no fue una barrera para realizar el procedimiento ni tampoco requirieron cuidados distintos a los que hubieran tenido si solo se le hubiera realizado la cirugía con anestesia general, beneficiándose del menor requerimiento de analgésicos posoperatorios. Tampoco vimos que tuvieran menor tiempo de duración del bloqueo del nervio periférico.

\section{Conclusión}

El presente estudio muestra que los BNPGE en PCH es un procedimiento seguro, que reduce los requisitos de los opioides y los efectos secundarios de ellos, mejorando el posoperatorio y la recuperación de los pacientes.

\section{Referencias}

1. Peyvandi F, Garagiola I, Young G. The past and future of haemophilia: diagnosis, treatments, and its complications. Lancet. 2016 Jul;388(10040):187-97. https://doi.org/10.1016/S01406736(15)01123-X PMID:26897598

2. Hemophilia. WFo. 2018; https://www.wfh.org/en/our-workresearch-data/annual-global-survey. Accessed October 2019

3. Sripada R, Reyes JJ, Sun R. Peripheral nerve blocks for intraoperative management in patients with hemophilia A. J Clin Anesth. 2009 Mar;21(2):120-3. https://doi.org/10.1016/j.jclinane.2008.06.030 PMID:19329016

4. Vanarase MY, Pandit H, Kimstra YW, Dodd CA, Popat MT. Pain relief after knee replacement in patients with a bleeding disorder. Haemophilia. 2007 Jul;13(4):395-7. https://doi.org/10.1111/ j.1365-2516.2007.01464.x PMID:17610555

5. Baker MD, Gullett JP. Ultrasound-Guided Femoral Nerve Blocks. Pediatr Emerg Care. 2015 Dec;31(12):864-8. https://doi. org/10.1097/PEC.0000000000000634 PMID:26626896

6. Tran DQ, Salinas FV, Benzon HT, Neal JM. Lower extremity re- 
gional anesthesia: essentials of our current understanding. Reg Anesth Pain Med. 2019 Jan;44(2):rapm-2018-000019. https:// doi.org/10.1136/rapm-2018-000019 PMID:30635506

7. Arnold C, Alvarado AC, Brady MF. Saphenous Nerve Block. Treasure Island (FL): StatPearls; 2020.

8. Marian AA, Ranganath Y, Bayman EO, Senasu J, Brennan TJ. A Comparison of 2 Ultrasound-Guided Approaches to the Saphenous Nerve Block: Adductor Canal Versus Distal Transsartorial: A Prospective, Randomized, Blinded, Noninferiority Trial. Reg Anesth Pain Med. 2015 Sep-Oct;40(5):623-30. https://doi. org/10.1097/AAP.0000000000000277 PMID:26110441

9. Krombach J, Gray AT. Sonography for saphenous nerve block near the adductor canal. Reg Anesth Pain Med. 2007 JulAug;32(4):369-70. https://doi.org/10.1016/j.rapm.2007.04.006 PMID:17720129

10. Saranteas T, Anagnostis G, Paraskeuopoulos T, Koulalis D, Kokkalis Z, Nakou M, et al. Anatomy and clinical implications of the ultrasound-guided subsartorial saphenous nerve block. Reg Anesth Pain Med. 2011 Jul-Aug;36(4):399-402. https://doi. org/10.1097/AAP.0b013e318220f172 PMID:21697687

11. Gray AT, Collins AB. Ultrasound-guided saphenous nerve block. Reg Anesth Pain Med. 2003 Mar-Apr;28(2):148. https://doi. org/10.1053/rapm.2003.50000 PMID:12677627

12. Chan VW, Perlas A, Rawson R, Odukoya O. Ultrasoundguided supraclavicular brachial plexus block. Anesth Analg. 2003 Nov;97(5):1514-7. https://doi.org/10.1213/01.
ANE.0000062519.61520.14 PMID:14570677

13. Neal JM, Gerancher JC, Hebl JR, Ilfeld BM, McCartney CJ, Franco $C D$, et al. Upper extremity regional anesthesia: essentials of our current understanding, 2008. Reg Anesth Pain Med. 2009 Mar-Apr;34(2):134-70. https://doi.org/10.1097/ AAP.0b013e31819624eb PMID:19282714

14. Brown DL, Cahill DR, Bridenbaugh LD. Supraclavicular nerve block: anatomic analysis of a method to prevent pneumothorax. Anesth Analg. 1993 Mar;76(3):530-4. https://doi. org/10.1213/00000539-199303000-00013 PMID:8452261

15. Morichika S, Shima M, Imanaka Y, Nakajima M, Iwasaki S, Yoshioka A. [Spinal canal bleeding in hemophilia A]. Rinsho Ketsueki. 1995 Jul;36(7):687-93. PMID:7563598

16. Allen HW, Liu SS, Ware PD, Nairn CS, Owens BD. Peripheral nerve blocks improve analgesia after total knee replacement surgery. Anesth Analg. 1998 Jul;87(1):93-7. PMID:9661553

17. Auroy $Y$, Narchi P, Messiah A, Litt L, Rouvier B, Samii K. Serious complications related to regional anesthesia: results of a prospective survey in France. Anesthesiology. 1997 Sep;87(3):47986. https://doi.org/10.1097/00000542-199709000-00005 PMID:9316950

18. de Boer HD, Detriche O, Forget P. Opioid-related side effects: postoperative ileus, urinary retention, nausea and vomiting, and shivering. A review of the literature. Best Pract Res Clin Anaesthesiol. 2017 Dec;31(4):499-504. https://doi.org/10.1016/j. bpa.2017.07.002 PMID:29739538 